\title{
Review of Recent Advances
}

THE ELECTROCARDIOGRAM AND THE POSITION OF THE HEART

\author{
Franklin D. Johnston, M.D., Joseph M. Ryan, M.D., ${ }^{*}$ \\ and J. Marion BRyant, M.D.
}

Ann Arbor, Mich.

$\mathrm{O}$ $\mathrm{NE}$ of the troublesome problems in the field of electrocardiography is to decide whether certain peculiarities in the tracings are due to abnormalities of the myocardium or to nothing more than an unusual position of the heart. Difficulties of this kind occur in connection with the interpretation of both limb and precordial leads and may limit the value of the records considerably. This paper is not a review of the extensive literature on the subject, but it is an attempt to point out some basic relationships between electrocardiograms and the anatomical position of the heart, to discuss some recent studies which have a bearing on the problem, and to emphasize some difficulties and unanswered questions arising from this work.

Einthoven, Fahr, and de Waart ${ }^{1,2}$ showed that the form of the standard electrocardiogram varies with respiration or change of posture and attributed this to a change in the position of the heart. Some years later, Meek and Wilson, ${ }^{3}$ working with dogs, reported consistent changes in the limb leads when the heart was rotated about the anteroposterior or the longitudinal axis alone and pointed out that, unless care is exercised, movement about the former axis is usually accompanied by rotation about the latter. Since the two types of rotation, so induced, act to shift the electrical axis in opposite directions, erratic and unpredicatable changes in the axis found in their early experiments were satisfactorily explained. These workers emphasized the importance of rotation of the heart on its long axis as a factor which determines the direction of the electrical axis and amply confirmed earlier studies by Groedel and Mönckeberg. ${ }^{4}$

It has been known for many years that standard electrocardiograms taken on normal subjects and on many patients with heart disease, often show changes if tracings are taken with the body in different positions. Thus, with the subject lying on the left side, a shift of the electrical axis to the right is usually seen, and a shift to the left may occur when the subject is lying on the right side. Alterations of this kind are particularly apt to be found in subjects with relatively

\footnotetext{
From the Department of Internal Medicine, University of Michigan Medical School, Ann Arbor. Some of the material in this paper was obtained with the help of a grant to Dr. Frank N. Wilson from the S. S. Kresge Foundation.

Received for publication Aug. 31, 1951.

*Voluntary Assistant in the Heart Station, July 1, 1950, to Jan. 1, 1951.
} 
vertical hearts, but the reverse may occur when the heart is transversely placed. In the first group it is obvious that these electrocardiographic findings are exactly the reverse of those one would expect if the heart rotated about the anteroposterior axis alone. They can only be explained by the predominant effects of accompanying rotation of the heart about the longitudinal axis. Not only may rotation of shall amount about this axis cause profound changes in the appearance of the limb leads, but unfortunately movement of this kind is very difficult to estimate accurately by other means. Roentgenographic studies are of limited value in the study of rotation of this type, and many currently accepted ideas about the position of the heart are based largely, if not entirely, on electrocardiographic data.

Dieuaide, ${ }^{5}$ in 1925, published standard electrocardiograms taken with patients lying alternately on the right and left sides. Striking differences in the tracings ordinarily caused by these changes of position were emphasized, and their absence in conditions, such as constrictive pericarditis, where the heart is fixed in the chest, was recognized and was pointed to as an aid in the diagnosis of these diseases. It is our impression that not enough attention has been paid to matters brought out by this early work, either as a guide in the diagnosis of conditions that may immobilize the heart or possibly as a tool for study of the relationships between the position of the heart and the electrocardiogram.

That change of posture does not always produce significant or consistent changes in the limb leads, even in the absence of constrictive pericarditis or allied conditions, was made clear by the studies of Nathanson ${ }^{A}$ in 1931. Sixty individuals, including normal subjects and patients with heart disease "in whom a fixed heart was improbable," were studied in the supine, left lateral, and right lateral positions. In fifty-five of these, definite electrocardiographic alterations with change in position were found, but in the remaining five individuals $(9 \mathrm{per}$ cent) tracings were similar in all three positions. The most marked and constant electrocardiographic changes were found when the subjects were placed in the left lateral position. Thus, shift of the electrical axis to the right in 83 per cent, shift to the left in 6 per cent, and no significant change in 11 per cent were found in this group. In the right lateral position, on the other hand, tracings were like those obtained in the supine position in 30 per cent of the subjects, while about one-half of the remainder showed a shift of the electrical axis to the left and one-half to the right. These results seem to indicate a considerable lack of correlation between body position and the electrocardiogram in subjects without disease known to immobilize the heart. Nathanson did not, however, analyze his results on the basis of the presence or absence of cardiac abnormality, nor did he mention the types of cardiac abnormality studied, except to state that individuals with hypertensive and coronary artery disease were included in the group.

It is obvious that electrocardiographic findings in limb leads with the subject in the supine position depend to a considerable degree on the position that the heart may have in the body, and if anything, such as change in posture or forced respiration, occurs to alter this position, modifications in these leads will appear. The situation is complicated because, even in normal subjects, the amount of 
rotation that can occur about three possible axes, the anteroposterior, the longitudinal, and the transverse, must depend on the motility of the mediastinal structures and the transverse and anteroposterior dimensions of the chest. Furthermore, simultaneous movement about all three axes may occur, and estimation of rotation about the latter two, except by inference from the electrocardiogram, is very difficult. When heart disease is present, abnormalities in the tracings may be exaggerated or be caused to disappear by shifts in the position of the heart. Some cardiac lesions lead to rotation particularly about the longitudinal axis, while others may prevent movement which might otherwise occur.

A recent paper by Brofman, Feil, Hellerstein, and Jones, ${ }^{7}$ showing that changes of posture may cause the appearance or disappearance of signs of old postcrior infarction in the limb leads, illustrates the difficulty that may exist in the interpretation of tracings taken from patients with known heart disease.

Wilson and co-workers, ${ }^{8}$ in 1941 , pointed out similarities of ten seen between the ventricular complexes of unipolar leads from the left arm and leg and those in tracings obtained from the right or the left side of the precordium in the same subject. On the basis of these observations, several positions of the heart defined entirely by electrocardiographic findings were recognized. Although it was felt by the authors that the electrocardiographic positions were in general related to the anatomical position of the heart, a necessary dependency of the former on the latter was not suggested, and caution in the interpretation of ventricular complexes of small voltage, especially those in tracings taken from points far from the heart, was advised. During the past ten years, Goldberger, ${ }^{9}$ Myers and Klein, ${ }^{10}$ and others, by studies which correlate the form of unipolar extremity tracings with records obtained by semidirect leads from the thorax or esophagus, have developed criteria by which it may be possible to evaluate rotation of the heart not only about the anteroposterior and longitudinal axes, but also about the transverse axis. Most of these proposals are logical, but how accurate some may be is a matter of upinion. These observations, like most other studies relating the electrocardiogram to the actual position of the heart, are supported almost entirely by electrocardiographic evidence.

The crux of the foregoing discussion, of course, revolves about the question: To what extent can one assume that the ventricular complexes in unipolar leads reflect the potential variations existing at the surface of the heart closest to the point from which the tracing was taken? Comparisons of direct and semidirect (precordial) leads taken during animal experiments make it clear that the QRS complexes seen in the latter closely resemble (except for size) those existing at the surface of the subjacent ventricle. Considerable evidence may be cited to indicate that the ventricular complexes in unipolar leads taken from regions quite distant from the heart are similarly determined. For example, the constancy with which QRS and $T$-wave changes due to posterior infarction appear in Lead $V_{F}$ seems to point to a clear dependency between the potential variations of the leg and those existing on the diaphragmatic surface of the left ventricle. Myers and Klein ${ }^{10}$ stated: "If sufficient precordial and esophageal leads are taken, a semidirect lead can be found in which the QRS-T pattern resembles that of a unipolar limb lead. 
The potential variations of an extremity are governed principally by those of the surface of the heart which faces toward the limb and thus depend upon the position of the heart in the chest."

It appears likely that the foregoing views are, in general, correct, but serious doubts of their validity may arise in some abnormal tracings where deflections in the unipolar extremity leads are small. In our experience, it is not unusual to find tracings where the ventricular complexes in neither Lead $\mathrm{aV}_{\mathrm{L}}$ nor $\mathrm{aV}_{\mathrm{F}}$ closely resemble those seen in records taken from the right or the left side of the precordium. Furthermore, occasional tracings are seen in which the electrocardiographic position of the heart changes within a short period of time where no reason for a shift in the position of the heart is present. This is illustrated by the

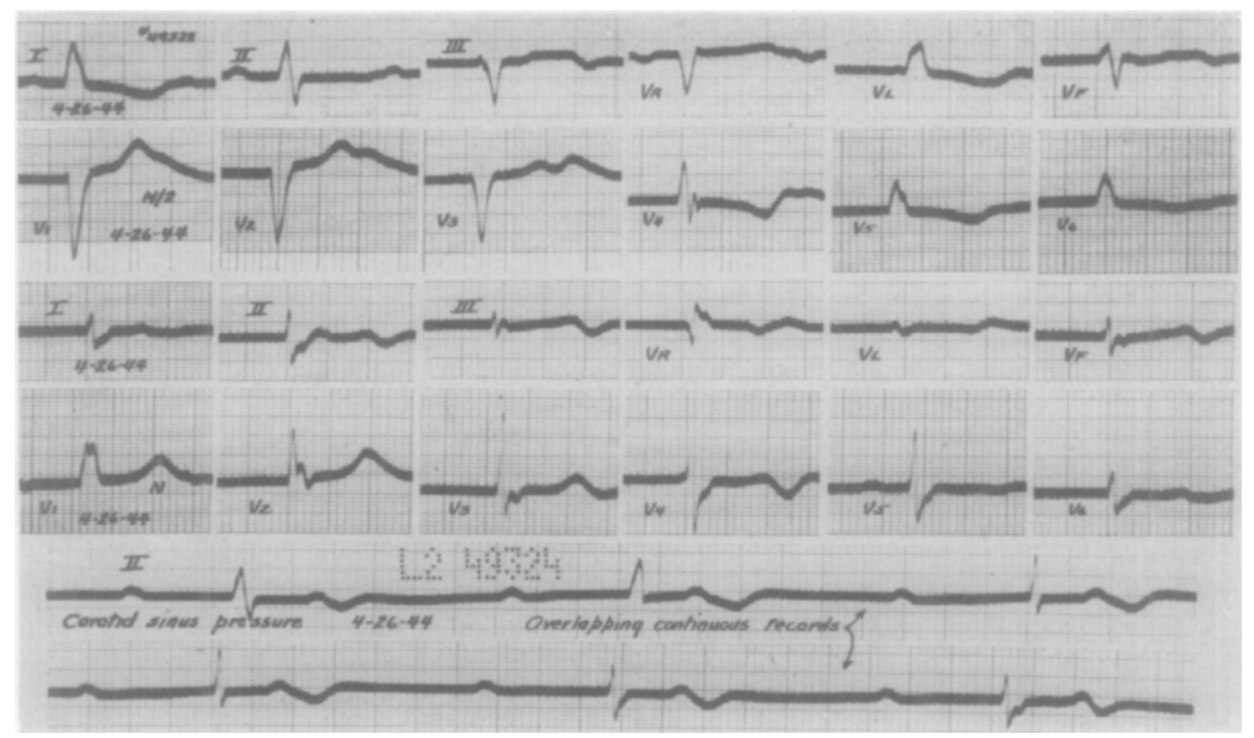

Fig. 1.-Tracings taken in rapid succession from an elderly patient with complete atrioventricular heart block. Spontaneous variation in the form of the ventricular complexes was present. Complexes of the type seen with left branch block are seen in the limb and precordial leads in the first and second rows. At this time the heart was in the horizontal position from the electrical standpoint. When complexes of the form seen in right branch block, shown in the third and fourth rows, were present, the heart was in a semivertical position. The two lower rows show a continuous strip of Lead II illustrating the change from one ty pe of complex to the other that could be produced by carotid sinus pressure. See text.

electrocardiograms reproduced in Fig. 1. These tracings were taken in rapid sequence on a man 83 years old who had arteriosclerotic heart disease with complete atrioventricular block. Ventricular complexes resembling those seen with left bundle branch block were present part of the time, but spontaneous change to a second type of complex like those seen in right branch block occurred. It was found that carotid sinus pressure would cause a transition from the first to the second type of complex. Such a change is seen in the continuous strip of Lead II shown in the lower part of Fig. 1. Of interest here is the fact that the electrocardiographic position of the heart changed from transverse to semivertical during the transition from one type of complex to the other. This is clearly shown in the four upper sets of tracings in Fig. 1. 
Studies on the spatial vectorcardiogram, particularly the work of Duchosal and Sulzer, ${ }^{11}$ Grant and Estes, ${ }^{12}$ and Conway, Cronvich, and Burch, ${ }^{13}$ involve concepts that may seem hard to reconcile with the commonly accepted interpretations of unipolar leads outlined previously. The latter emphasize the importance of potential variations existing at the surface of various aspects of the heart, whereas the spatial vectorcardiogram is based on the assumption that the electrical activity of the heart as a whole may be represented by a series of vectors each of which indicates the direction and magnitude of the average or resultant electrical force acting within the heart at any instant. The termini of these vectors establish the figures known as vectorcardiograms. If one remembers that the methods now available for obtaining spatial vectorcardiograms are based on the proper temporal combination of figures obtaincd from more or less conventional surface leads in two different planes, it should be clear that the vector curves are simply a different and perhaps more informative way of expressing information usually presented in ordinary bipolar or unipolar leads. Until a more accurate and independent method for determining the direction and magnitude of voltages produced within the heart is developed, one cannot say that any basic conflict between the interpretation of vectorcardiograms and of usual surface leads exists.

These comments about spatial vectorcardiograms must not be construed as minimizing the value of work that has been done in this special field, since this is not true. As Grant and others have pointed out, all surface leads may be derived from the spatial vectorcardiogram by projection, and therefore studies which lead to its proper construction, understanding, and use may greatly simplify electrocardiographic diagnosis and teaching. Furthermore, when techniques are available for the quick and accurate estimation of these spatial curves, most current problems, concerned with peculiarities in the position of the heart, will cease to exist, because differentiation between normal and abnormal records will be made largely on the size and form of the vectorcardiograms themselves and the relations existing between various components of the spatial figures. Unusual orientation of a normal spatial complex due to a shift in the anatomical position of the heart would be readily apparent, and peculiarities in derived surface leads would be easily explained. In spite of the information to be gained by the use of vectorcardiograms, it is unlikely that these records will replace tracings of the conventional type in the near future. The many unanswered questions relative to the proper technique for obtaining spatial vectorcardiograms and the expensive and complicated apparatus required place vectorcardiography definitely in the field of research at the present time.

Gardberg and Ashman, ${ }^{14}$ in a thoughtful paper, have tried to explain changes in the standard leads occurring with rotation of the heart, especially about the anteroposterior and longitudinal axes. On the basis of knowledge of the general course of the activation wave in th? hearts of experimental animals and other data, a series of vectors in three-dimensional space representing the resultant voltages acting at a number of instants during the period of ventricular activation is described. When this series of vectors is used throughout, the character of QRS complexes that may be expected to occur in the standard leads with the 
heart in various positions about the anteroposterior and long axes is clearly shown in Figs. 9 through 12 of their paper. Effects of movement of the heart about the transverse axis are also discussed briefly, and in a subsequent paper by Ashman ${ }^{15}$ the electrocardiographic changes due to rotation of this type are considered in greater detail. Referring again to the figures published by Gardberg and Ashman, one is surprised by the large amount of rotation of the heart about the longitudinal axis which the authors believe may occur normally. This is particularly striking in their Fig. 12, which illustrates marked clockwise rotation about this axis. Here, some forces, arising primarily in the free wall of the left vencricle, are so greatly altered in direction that their projections on the frontal plane point from left to right rather than in the opposite direction. If this situation exists in the normal subject, it may occur to an even greater degree in patients with right ventricular hypertrophy or dilatation. Kossmann, Berger, Brumlik, and Briller $^{16}$ have indeed suggested that right axis deviation in the limb leads and large $\mathrm{R}$ waves found in precordial tracings taken from the right side of the precordium in individuals with marked hypertrophy of the right ventricle are rarely due to the enlarged right ventricle but to marked clockwise rotation of these hearts about the longitudinal axis and "almost complete reversal of the electrical fields of the two ventricles." This work challenges some of the generally accepted ideas relating to interpretation of precordial leads. 'This is particularly true since it is based largely on results of intracardiac electrocardiographic studies which appear to indicate, among other things, that electrical forces across the free wall of the right ventricle, even when it is hypertrophied, may be small. $R$ waves of nearly the same size and with peaks occurring simultaneously, or nearly so, have been found in tracings obtained from the cavity of the right ventricle and from the chest wall as close as possible to the location of the intracavitary electrode by Kossmann ${ }^{16}$ and others. This suggests, of course, that the $\mathrm{R}$ waves in question are due largely to forces passing from left to right through the interventricular septum. Rosenman ${ }^{17}$ emphasized this fact and quite correctly pointed out that activation of the free wall of the right ventricle may occur during the downstroke of the $\mathrm{R}$ wave (the intrinsicoid deflection). Wilson and co-workers ${ }^{18}$ discussed this matter earlier and showed why the end of the intrinsicoid deflection rather than its beginning (peak of $R$ wave) must signal the arrival of the activation wave at the epicardial surface of the ventricle beneath the electrode.

Before these matters are considered further, some other perplexing problems relating to the interpretation of precordial leads must be mentioned. In the majority of subjects with normal hearts and patients with heart disease, the usual sites for placement of the exploring electrode are considered satisfactory, since the first two or three overlie the right ventricle and those located farther to the left are relatively close to the left ventricle. Furthermore, a line connecting the stations on the chest crosses the plane of the interventricular septum in a nearly perpendicular fashion so that the transitional zone is a narrow one. When peculiarities in the ventricular complexes in usual multiple precordial electrocardiograms, such as broadening of the transitional zone, displacement of this region to the right or left, or failure to obtain tracings of the left (or right) ventricular type, are observed, one naturally suspects an unusual position of the heart, 
but reliable evidence from other sources to confirm such a view may be scanty or absent. Additional tracings taken at higher or lower levels or farther to the right or left than usual may help to explain peculiarities in the routine multiple precordial leads, and such additional studies should be obtained if it is possible. It has been suggested that the usual sites for location of the exploring electrode be altered to conform with the anatomical position of the heart in instances where the latter is suspected or is known to be unusual. At the moment such a policy would appear to be unwise, since it would add another variable to a technique which is already sufficiently complicated.

In the interpretation of precordial leads, it is generally assumed that the subendocardial tissues become electrically active very early and that the QRS complex is largely due to forces that pass in a simple radial fashion from the endocardial to the epicardial surfaces of the ventricles. The interventricular septum is believed to be activated from both sides, although considerable evidence suggests that in most human subjects with normal intraventricular conduction activation starts somewhat earlier on the left side than it does on the right. An upward deflection of the QRS complex is considered to indicate predominance of electrical forces passing toward the exploring electrode, while a downward deflection suggests that those moving away from the electrode dominate. Used with some reservations, these concepts have been of great value in the explanation of changes in the QRS complex in bundle branch block, myocardial infarction, hypertrophy, and some other conditions. Nevertheless, some findings in precordial tracings are hard to understand if one assumes the simple type of spread of the activation wave outlined.

In many normal precordial electrocardiograms, in some showing changes due to left ventricular hypertrophy, and in most records with complete left bundle branch block, $\mathrm{S}$ waves in tracings from the right side of the precordium are considerably larger than are the $\mathrm{R}$ waves in tracings from the left side. This finding is particularly striking and common in the latter group of records, and it is rather difficult to explain. One might expect the opposite situation to exist, since $\mathbf{S}$ waves seen in tracings obtained from the right side of the precordium presumably originate from regions of the heart some distance away from the exploring electrode, whereas the $\mathrm{R}$ waves in records from points over the left precordium must arise to a large extent in the free wall of the left ventricle, which is relatively close to the electrode. The difficulty here may be resolved if one assumes that the activation wave does not always travel directly outward from the endocardial to the epicardial surface of the ventricle but may spread tangentially rather than radially over parts of the ventricle. Proof for this idea is lacking, and it must be admitted that a departure from the concept of purely radial spread introduces complications to the interpretation of precordial leads.

Little attention has been paid to the muscle bundles, known to exist in the ventricles, as important factors in determining the spread of the activation wave over these chambers, but, if one considers tangential spread as a possibility, these anatomical units may play an important role. Robb and Robb, ${ }^{19}$ a number of years ago, suggested that the activation wave may follow the course of the muscle 
spirals, but no clear evidence supporting this view has been presented, and most workers have considered the ventricles to behave as a syncytium from the electrical standpoint. Recent studies which suggest that the free wall of the right ventricle, even when it is hypertrophied, contributes very small voltages during activation should be reviewed with the possibility of tangential spread of the electrical wave in mind. Close similarities in tracings obtained by a lead from the cavity of the right ventricle and one on the precordium as close as possible to the intracavitary electrode suggest that the impulse may not pass in a simple radial fashion through the free wall of the right ventricle and that tangential spread may exist. Further speculation regarding these basic matters is hardly worth while, but careful experiments, designed to answer the questions raised, are clearly needed.

Returning to the suggestion of Kossmann and co-workers ${ }^{16}$ that signs of right ventricular enlargement in both $\operatorname{limb}$ and precordial leads may be due to marked clockwise rotation of the heart about its long axis, we wish to point out one fact which argues against their proposal. Right ventricular enlargement often occurs with right bundle branch block, and, under these circumstances, large, late $R$ waves are regularly found in tracings from the right side of the precordium, including records obtained from points 3 and 4 on the right side of the chest $\left(V_{3 R}\right.$ and $\left.V_{4 R}\right)$. Any argument that the large, late $R$ waves arise in the left ventricle here is clearly untenable.

McGregor ${ }^{20}$ has recently reported that electrocardiograms taken from the surface of the heart during operations on patients with the tetralogy of Fallot show relatively small $R$ waves and large $S$ waves in the tracings taken with the exploring electrode in close proximity to the right ventricle and $R$ waves that dominate the QRS complex in the records taken close to the left ventricle. These observations appear to support the view that large $R$ waves found in tracings taken from the right side of the precordium in patients with right ventricular hypertrophy may arise from the left ventricle of a markedly rotated heart and thus support the suggestion of Kossmann and co-workers that clockwise rotation of the heart about the long axis and not hypertrophy of the right ventricle produces the large $\mathrm{R}$ waves in question. In these studies, however, the pericardium was not opened as a rule, and the part of the right ventricle lying nearest to the exploring electrode was probably somewhat uncertain. It would not be surprising to obtain tracings in which a small $R$ wave and large $S$ wave appear if the electrode were placed close to the trabeculated area of the right ventricle, and further studies with wider exploration of the hypertrophied right ventricle and more exact localization of the exploring electrode on this chamber must be carried out. If it is found that the hypertrophied right ventricle does not produce the large $\mathrm{R}$ waves found in records from the right side of the precordium, the reasons for its apparent lack of electrical activity must be determined. In this connection, the possibility of tangential spread of the activation wave rather than primarily radial penetration through the free wall of this chamber must be investigated. 


\section{SUMMARY}

The profound effects that rotation of the heart, particularly about the longitudinal axis, have on the mean electrical axis, as seen in limb leads, are emphasized, and some of the experimental and clinical studies that bear on this matter are discussed. The advantages that spatial vectorcardiograms may have in the evaluation of electrocardiographic changes due solely to an unusual position of the heart are pointed out, but, for a number of practical reasons, it is felt that such records will not be very useful in the solution of these or similar problems in the near future.

Peculiarities in the form of tracings from the precordium due entirely to an unusual position of the heart are mentioned, but it is considered unwise (except for purposes of investigation) to recommend a change from the conventional sites for multiple precordial leads because of the suspicion that the heart may be placed in an unusual fashion.

The suggestion of Kossmann and co-workers that signs suggesting right ventricular enlargement in both standard and precordial leads may arise as a result of marked clockwise rotation of the heart about the long axis is discussed, and some evidence for and against this view is presented. Since concepts basic in the interpretation of precordial leads are involved in this problem, the need for further studies to decide the matter is emphasized. It is pointed out that tangential rather than purely radial spread of the activation wave over the ventricles may explain some of the difficulties encountered in interpretation of precordial leads, particularly when right ventricular enlargement or left bundle branch block is present.

\section{REFERENCES}

1. Einthoven, W., Fahr, G., and de Waart, A.: On the Direction and Manifest Size of the Variations of Potential in the Human Heart and on the Influence of the Position of the Heart on the Form of the Electrocardiogram, Pflüger's Arch. f. d. ges. Physiol. 150: $275,1913$.

2. Ibid., translated into English by Hoff, H. E., and Sekelj, P., AM. Heart J. 40:163, 1950.

3. Meek, W. J., and Wilson, A.: The Effect of Changes in Position of the Heart on the QRS Complex of the Electrocardiogram, Arch. Int. Med. 36:614, 1925.

4. Groedel, T., and Mönckeberg, J. G.: Ein Fall von Kongenitaler Pulmonalstenose und die sich daraus ergebenden Schlüsse für die Initialzacke des Electrokardiogrammes, Zentralbl. f. Herz- u. Gefässkr. 5:2, 1913.

5. Dieuaide, F. R.: The Electrocardiogram as an Aid in the Diagnosis of Adhesive Pericardial Mediastinitis, Arch. Int. Med. 35:362, 1925.

6. Nathanson, M. H.: Electrocardiographic Study of Movements of the Heart With Change of Posture, Proc. Soc. Exper. Biol. \& Med. 28:766, 1931.

7. Brofman, B. L., Feil, H., Hellerstein, H. K., and Jones, A. M.: Postural Electrocardiographic Changes in Healed Myocardial Infarction, Circulation 3:752, 1951.

8. Wilson, F. N., Johnston, F. D., Cotrim, N., and Rosenbaum, F. F.: Relations Between the Potential Variations of the Ventricular Surfaces and the Form of the Ventricular Electrocardiogram in Leads From the Precordium and the Extremities, Tr. A. Am. Physicians 56:258, 1941.

9. Goldberger, E.: Unipolar Lead Electrocardiography, ed. 2, Philadelphia, 1949, Lea \& Febiger.

10. Myers, G. B., and Klein, H. A.: The Relation of Unipolar Limb Leads to Precordial and Esophageal Leads, AM. HEART J. 35:727, 1948.

11. Duchosal, P. W., and Sulzer, R.: La Vectocardiographie, Bâle, 1949, S. Karger.

12. Grant, R. P., and Estes, E. H., Jr.: Spatial Vector Electrocardiography, Philadelphia, 1951, The Blakiston Company. 
13. Conway, J. P., Cronvich, J. A., and Burch, G. E.: Observations on the Spatial Vectorcardiogram in Man, Ам. HeART J. 38:537, 1949.

14. Gardberg, M., and Ashman, R.: The QRS Complex of the Electrocardiogram, Arch. Int. Med. 72:210, 1943.

15. Ashman, R.: Estimation of the Heart Position From the QRS Complex of the Electrocardiogram, Arch. inst. cardiol. México 16:139, 1946.

16. Kossmann, C. E., Berger, A. R., Brumlik, J., and Briller, S. A.: An Analysis of Causes of Right Axis Deviation Based Partly on Endocardial Potentials of the Hypertrophied Right Ventricle, Am. HEART J. 35:309, 1948.

17. Rosenman, R. H.: Observations on the Genesis of the Electrocardiogram, AM. HEART J. 40:522, 1950.

18. Wilson, F. N., Rosenbaum, F. F., and Johnston, F. D.: Interpretations of the Ventricular Complex of the Electrocardiogram, Advances Int. Med. 2:1, 1947.

19. Robb, J. S., and Robb, R. C.: The Normal Heart, Anatomy and Physiology of the Structural Units, AM. HEAR'T J. $23: 455,1942$.

20. McGregor, M.: The Genesis of the Electrocardiogram of Right Ventricular Hypertrophy, Brit. Heart J. 12:351, 1950. 